\title{
Ship extraction and categorization from ASTER imagery
}

\author{
Panagiotis Partsinevelos ${ }^{1}$, George Miliaresis ${ }^{2}$ \\ ${ }^{1}$ School of Mineral Resources Engineering, Technical University of Crete, \\ Chania, Greece, Email: ppartsinevelos@isc.tuc.gr \\ ${ }^{2}$ Open University of Cyprus, Faculty of Pure \& Applied Sciences \\ Email: miliaresis.g@gmail.com
}

\begin{abstract}
:
The detection and parametric representation of objects from satellite imagery within the sea environment is of big importance for recording both static (small rocky islands and reefs) and dynamic objects (ships). Applications in environmental protection and safety include ship related pollution such as oil slicks, sea traffic, coastal management, fishery monitoring, etc. In this study, a ship extraction and categorization methodology is presented from low resolution (applicationwise) multispectral ASTER imagery, corresponding to the sea region south east of Athens in Greece. At a first level, in the radiometrically corrected image, quad tree decomposition and bounding rectangular extraction automatically outline location of objects - possible ships, by statistically evaluating spectral responses throughout the segmented image. Subsequently, the object borders within the rectangular regions are extracted, while connected component labelling combined by size and shape filtering allows for ship characterization. The ships spectral signature is determined in green, red and infrared bands while cluster analysis allows the identification of ship categories on the basis of their size and reflectance. Additional pixel based measures reveal estimated ship orientation, direction, movement, stability and change in direction. The results are complemented with additional geographic information and inference tools are formed towards the determination of probable ship type and its destination.
\end{abstract}

Keywords: ASTER imagery, ship detection, image segmentation,

\section{Originality and Contribution}

The aim of this research is to present a method for ship extraction, recognition, categorization and dynamic information inference from ASTER VNIR imagery. The novelty of the paper is described as follows:

- This is the first attempt to the best of our knowledge for ship extraction through ASTER imagery.

- The major challenge is to derive information from medium resolution images compared to ship size and shape.

- The image processing based methodology presented, involves algorithmic variations, including a first step generalized radiometric segmentation, automated thresholding criteria, and shifted rectangle formation, to compensate for regionally differentiating sea illumination and avoid non-ship objects.

- The combination of the image processing ship categorization with geographic information provides inference results, including possible destination and ship type from a single snapshot.

Overall, the novelty is summarized as the deduction of dynamic information and inference from a static image. Application-wise, image exploitation of modern, medium resolution, multispectral satellite imagery, impacts the fields of ship traffic monitoring with probable applications in environmental protection and safety. Such an effort also aids in expected behavior and type classification of ships. It could serve as a parallel process beyond SAR oriented ship extraction, which is prone to noise and wave formations. Based on satellites' revisiting periods which vary according to their orbits, dynamic information from stable snapshots reveals tendencies and fills temporal gaps. The presented methodology proves readily applicable to satellite imagery of relevant resolution, and is customable to satisfy additional resolutions under minor alterations. 


\section{INTRODUCTION}

Among an extensive collection of applications, multispectral satellite imagery is used for recoding biophysical parameters and monitoring the ocean and coastal environment [1-4]. The detection and parametric representation of objects from satellite imagery within the sea environment is of big importance for recording both static (small rocky islands and reefs) and dynamic objects (ships). Other applications include ship related pollution such as oil slicks, sea traffic [5], coastal management, fishery monitoring, and defense [6].

Nowadays micro-satellite platforms are equipted with a combination of both active (microwave) and passive (multispectal) sensors [7]. Microwave remote sensing systems (Seasat, ERS-1, JERS1, ERS-2 and RADARSAT-1) have been extensively used for ship detection [8-10].

In the multispectral area, ship detection and further classification based on type and size requires very high resolution imagery $[11,12]$ and it provides a relatively even sea reflectance close to black. Accordingly, image pixel patches of ships provide large traces for ship categorization [13]. The dynamic nature of sea traffic is most of the times addressed through multi-temporal imagery forming sea traffic maps [5]. Generally speaking, SAR oriented procedures are mainly used even though they are prone to noise and wave formations, while passive systems are vulnerable to various weather conditions.

Advanced Spaceborne Thermal Emission and Reflection Radiometer (ASTER) sensor, emerged from a cooperative effort between NASA and Japan's Ministry of International Trade and Industry [14] that obtains high resolution (15 to $90 \mathrm{~m}$ ) images of the Earth in the visible, near-infrared (VNIR), shortwave-infrared (SWIR), and thermal infrared (TIR) regions of the spectrum. ASTER data products include: spectral radiance and reflectance of the Earth's surface, surface temperature and emissivity, digital elevation maps from stereo images, surface composition and vegetation maps, cloud, sea ice, and polar ice products, etc $[15,16]$. The VNIR subsystem operates in three bands (green, red and near-infrared) with $15 \mathrm{~m}$ resolution and a $60 \mathrm{~km}$ swath width [17]. The revisiting period is about 20 days.

The aim of this research is to present a methodology for ship extraction, recognition, categorization and dynamic information inference from ASTER VNIR imagery exploring its applicability with respect to ship detection. Such an effort aids the ship related applications such as sea traffic, expected behavior, ship type classification, etc. The challenges include low resolution processing according to the size of the ships, deduction of dynamic information from a static image and formation of inference tools.

\section{METHODOLOGY}

Ship detection and categorization methodology are discussed in the remaining of the paper through the following steps:

1. Preprocessing of the satellite imagery including destriping and reflectance value estimation.

2. Quad tree decomposition - segmentation of the image compensating for varying sea reflectance and targeting for the subsequent image processing algorithms.

3. Possible ship region extraction through bounding rectangular patches based on background sea and ship reflectance, prone to non-ship object inclusion.

4. Ship detection and extraction from the rectangular regions and further categorization based on shape and reflectance.

5. Additional ship measures and classification of dynamic nature.

6. Inference logic through integration of additional geographic related information.

\subsection{Study area and data}

The satellite imagery was acquired on the 11th of December 2001 from the US Geological Survey during the one-year evaluation period of ASTER characterized by processing level 1A (no radiometric and geometric corrections were applied). The latitude and the longitude are within the range 37.4878 to 38.1428 and 23.1977 to 24.0596 respectively. 


\subsection{Pre-processing of ASTER imagery}

First, radiometric corrections were applied. Each band was destriped using the histogram matching technique and digital values were converted to radiance on the basis of the corresponding band gain [18]. The ASTER imagery was georeferenced and geometrically corrected by the use of orbital data available in the image header (figure 1). The very near infrared band (band 3) was selected for ship detection due to the very low reflectance of water bodies in the infrared portion of spectrum [19].

\subsection{Sea region decomposition}

Quad tree based decomposition [20, 21] divides the sea region into sections which are tested for the uniformity of their spectral response (figure 2). For each of the segmented sub-images the average and standard deviation of grayscale values are estimated. This procedure serves two objectives selectively. First, the ship detection algorithm is interrupted if the standard deviation is low, indicating uniformity in the sea and thus avoiding time consuming processing. Second, since sea illumination may slightly vary throughout the image, uniform zones of the sea surface regions are used to define the background sea thresholds for the next steps, and thus contemplating for local spectral attitude.

\subsection{Ship region targeting}

Upon the selected quad regions the objective of this step of the procedure is to further delineate a vague generalized region of possible ship position, in order to target the subsequent image processing procedures. The algorithm initiates by segmenting the sea quad regions into ordered rectangles of constant dimensions, where pixel intensity counts are computed. These bounding rectangles are examined as of the population of contained pixels with gray values over a threshold, indicating possible ship existence.

The arbitrary distribution of the bounding rectangles may divide a single ship in up to four parts and thus diminish the population of indicative ship pixels in each rectangle, making the algorithmic computation highly sensitive to rectangle initiation and further placement. Thus, a routine is devised to iteratively segment the sea region under identical rectangle size, but incrementally shifted in $\mathrm{x}$ and $\mathrm{y}$ axes. The overall generalized ship occupancy is computed through the union of the regions under the shifted routines (figure 3 ).

Throughout the described process, a series of thresholds and design variables are specified in order to reach a confident result. All these variables are automatically selected based upon the image scale, corresponding pixel size and spectral response statistics. More specifically, for the pixel size of 15 meters and the region under consideration, ship sizes vary from a few meters to 150 meters (in average), corresponding to 1 by 10 pixels. This average size may be increased on moving ships due to water turbulence also known as wake [22, 23]. Thus, the lower threshold of pixel population, indicative of a ship's existence is defined as around 8 pixels. Pixel population thresholds do not hold only for lower but also for upper limits in order to avoid small land areas where pixels over the threshold are highly populated (figure 4 right). This upper limit is selected as a portion of the bounding rectangle size. In order to acquire safe measures, the selected bounding rectangle size is considered around 300 by 300 meters corresponding to 20 by 20 pixels, based upon a maximum ship size.

Additional values that need to be automatically determined are the illumination thresholds over which the pixels are considered as ship parts. To estimate these thresholds for each of the quad regions the medium sea illumination is estimated. Ship illumination density is expected as very high compared to the sea region. The sea illumination standard deviation is added since ship response is expected to lie on the outer high parts of the distribution. The efficiency of the algorithm even under stripped imagery is evident in figure 4 left. Nevertheless, some false positive hits are apparent but are eliminated during the consequent processes. 
Moreover, in a customizable ship extraction procedure if one needs to find a priori all the ships in a specified direction, the bounding rectangles can be selected under a certain orientation size e.g. of 3 by 20 pixels for East-West direction.

\subsection{Ship Extraction}

The ship's border is outlined by a high pass filter, since an increased slope in the reflectance should be observed in the transition from sea to the ship pixels. The partial derivatives along the horizontal (x) and the vertical (y) direction are computed in a kernel of size $3 * 3$, by the SOBEL operator [19] and the slope is estimated [24]. The slope is expressed in degrees in the interval from $\left[0\right.$ to $\left.73^{\circ}\right)$. The pixels within the rectangular regions of figure 3 present slope in the interval $[0$, $55^{\circ}$ ). The slope image detail (derived from VNIR band 3) is presented in figure 5 left. Note that only the border region of the ships is excluded, since the pixels of the inner portion appear to preserve smoother reflectance variations (slope approaches zero).

The frequency histogram of the slope pixels within the rectangular regions of figure 5 left, is presented in figure 5 middle. Thresholding is applied to the slope image. A slope threshold is identified through a trial and error procedure. The pixels with slope greater than $3^{\circ}$ were labelled as ship border pixels. Note that occasionally the inner ship pixels (figure 5 right) failed to be segmented by thresholding since they represent slope values less than $3^{\circ}$. These small islands of background pixels surrounded by foreground pixels (ship border), were identified by a connected component labelling algorithm [25] and merged to the surrounded pixels [26]. Finally, 107 objects are identified. These objects are parametrically represented on the basis of size (expressed in pixels), and their mean reflectance in ASTER VNIR bands 1, 2 and 3 (Table 1). In the following section these objects are classified into 5 clusters (Table 1).

\subsection{Ship clustering}

The spectral signature of the ships in ASTER VNIR imagery (green, red and infrared channels) and their size are used for the identification of ship groups with almost similar parametric representation by means of cluster analysis.

Cluster analysis is a multivariate procedure which is commonly used for regional classification. It is based on some measurement of distance among objects, (for example Euclidean distance), which is calculated in a c-dimensional space, where $\mathrm{c}$ represents the number of attributes used in the clustering process $[19 ; 27]$. The centroid method employed, requires a priori definition of the number of clusters. The results for 5 clusters are shown in Table 2 and figure 6.

In order to evaluate the existence of statistically significant differences in the distances among the cluster centers, the statistical technique of one-way analysis of variance (ANOVA) was used. ANOVA calculates the between-cluster mean square and the within-cluster mean square. The ratio of these two mean squares is the usual ANOVA F statistic (Equation 1).

$$
F=\frac{\text { Variation between groups sum of squares } / k-1}{\text { Variation within groups sum of squares } /(N-k)}
$$

where, $\mathrm{k}$ is the number of groups and $\mathrm{N}$ is the total sample size.

The logic of the test argues that for the class (cluster) means to be significantly different, interclass (between cluster) variance must be of much greater magnitude than intraclass (within clusters) variance $[28 ; 29]$.

The results of the ANOVA procedure for the each centroid are shown in Table 3. Although the significance levels for the F statistic are below .001, the values of $\mathrm{F}$ in the case of cluster analysis should be mostly interpreted in terms of comparing differentiation of the cluster means for 
individual variables [27]. An alternative way to assess cluster differentiation is to derive the centroid distances among pairs of clusters (divergence) as it is indicated in Table 4.

\subsection{Ship dynamic information categorization and inference}

Based on the extracted ships from the previous step (figure 7a), a series of additional processes are performed towards ship categorization under various criteria. These procedures determine dynamic - time related information from a static image and are prone to subjectivity.

Beyond size, a general shape measure is derived from the elongation - eccentricity of the object in question, in order to determine whether it is a ship or e.g. a platform. For the specific image scale, it is highly unlike to discern the rear from the front of the ship [13]. Based on the assumption that the ship's wake would be a thin tail of high reflectance (figure 7a and c), the ship's course line can be automatically determined. In addition, each ship's minimum bounding rectangle (MBR) is determined and orientation-azimuth is estimated through the principal eigenvector of the ship pixels (figure 7e).

Three different ways for the determination of possible orientation are devised. First, the displacement between the center of gravity and center of the MBR reveals the possible orientation of the ship's heading (figure 7f), since the wake's trace would be thinner than the actual ship. (eccentricity). Second, two snapshots (figure b and d) of the ship using different grayscale thresholds indicate the direction as seen in figure $7 \mathrm{~g}$. Finally, assuming a bright ship compared to a less bright wake, the concentration of bright pixels in one side implies the direction (figure $7 \mathrm{~h}$ ). As stated before the scale is prohibiting a large extent of confidence in such processes. Nevertheless, these three direction measures enhance the overall determination confidence (figure 9a). In the case that the actual area of the ship-wake snapshot compared to the convex hull area is relatively small turning of the ship is implied as seen in figure 8.

Up to this point, ship related information is summarized as: general shape, size, stability, mobility, turning, course line, orientation. Inference procedures can be created in a GIS environment using the measures discussed so far complemented by additional information. Towards this objective we form the following inference tools:

- Small static ships (size) close to the shore (distance to the shore) and of relatively high population (density measure) can be considered as fishing ships [30], as seen in figure 9c on the Northern area, pointed with red circles.

- The ship's course directions provide an estimation of the ports' expected traffic (figure $9 \mathrm{~b}$ ) and may reveal natural or local ports as seen on figure $9 \mathrm{c}$.

- Lines of direction for non turning ships, away from the shore, and aligned to the digitized ship routes acquired from the ferries' companies (red dotted lines in figure 9c) along with the ship size can reveal possible destination, even if the destination is not included in the images. Categorization of destinations for the study area may include the island of Crete (figure 9c) or Islands on Central Aegean or even local.

\section{DISCUSSION OF RESULTS}

Bounding rectangular selection provided an initial approximation of possible ship location and since SOBEL was applied in the bounding rectangles, the effect of local high frequency noise in seed selection was reduced. On the other hand, the SOBEL operator allowed a data driven (thresholding of the slope frequency histogram on the basis of a trial and error procedure) seed pixel identification. The slope threshold is a function of both the a) physical conditions of sea (waves, currents, winds) during the satellite image acquisition and the b) the spectral resolution of the image band selected. 
There is one more limit, posed by the spatial resolution of satellite imagery ( $15 \mathrm{~m}$ in the current case study). Pixel size specifies the minimum size of objects that might be recognised. Since the background (sea) presents a rather uniform spectral response, ships might be recognised if their size is smaller than the pixel size, assuming that mixed pixels might present a different overall reflectance than the pure sea pixels.

Ship surfaces do not present a uniform reflectance, although the reflectance difference in border pixels is the key factor for ship delineation. This is not really a problem since the inner pixels are merged to the border pixels by a connected components pixel labelling algorithm and a size threshold. The ship shape is difficult to be derived accurately due to the $15 \mathrm{~m}$ pixel size (evidence of mixed pixels) in comparison to the rather moderate resolution ship size in the study area.

The spectral classification of ship objects on the basis of the ASTER VNIR bands (green, red and very near infrared) identified 3 groups of objects. The smallest in size objects present highest reflectance in band 2 (red) while the maximum in size object present the highest reflectance in band 3 (very near infrared). We can not verify whether this effect is due to mixed pixel effect (more severe for small in size ship) or the different spectral signature of the larger ships (the great in size oil tankers possibly present a different spectral response than the small in size cargo ships).

Multitemporal and very high resolution imagery along with combined SAR and multispectral processes are by far the desired means to acquire safer measures for ship extraction and related applications. But even the relative low resolution of a static ASTER image provided aiding measures and dynamic information.

\section{CONCLUSIONS - FUTURE WORK}

The discussed procedure for the detection and categorization of ships from ASTER VNIR imagery seems to be capable of ship pixel detection and delineation combined by connected components labelling and size thresholding. Limitations are posed by the pixel size $(15 \mathrm{~m})$ and spectral resolution of the VNIR ASTER imagery (green, red and very near infrared). The spectral signature of the ships in ASTER VNIR imagery (green, red and infrared bands) was determined while cluster analysis allowed the identification of ship categories on the basis their spectral response. These categories are shape and size dependent, indicating either the effect of mixed pixels or that the greatest in size ships are of different type. In addition, further categorization of dynamic nature and inference procedures proved the versatile applicability of the discussed processes.

In the future a combined SAR and ASTER processing should take place in order to combine the advantages of each platform, namely weather independence and sea reflectance uniformity. Additional inference tools combined with geographic and other information should be devised and tested in a GIS environment. Finally, fusion of imagery and information addressing the temporal aspect of ship related applications, will further enhance the research potential and applicability. 


\section{REFERENCES}

1. Frazier P., Page K. (2000) Water body detection and delineation with Landsat TM data. Photogrammetric Engineering and Remote Sensing, 66(12), 1461-1467.

2. Zhang Y. (2000) A method for continuous extraction of multispectrally classified urban rivers. Photogrammetric Engineering and Remote Sensing, 66(8), 991-999.

3. Neville R., Coward R., Watson R., Inglis M., Morain S. (2000) The application of TM imagery and GIS data in the assessment of arid lands water and land resources in west Texas. Photogrammetric Engineering and Remote Sensing, 66(11), 1373-1379.

4. Mohd I., Seeni M. (1989) Water depth determination from satellite data. ACRS, session in Oceanography, http://www.gisdevelopment.net/aars/acrs/1989/F/ocean002.shtmlACRS 1989

5. Hajduch G., Kerbaol V. (2006) Ships detection on ENVISAT ASAR data: results, limitations and perspectives, SEASAR, ESA/ESRIN, Frascati.

6. Zhang, F., Wu, B. (2008) A scheme for ship detection in inhomogeneous regions based on segmentation of SAR images. International Journal of Remote Sensing, 29(19), 5733-5747.

7. Høye G., Eriksen T., Narheim B., Wahl T. (2001) Global Fisheries Monitoring from Small Satellites. 3rd IAA Symposium on Small Satellites for Earth Observation, IAA-B3-0804.

8. Vachon P., Olsen R. (1998) RADARSAT SAR Mode Selection for Marine Applications: Amendments Based on Post-Launch Experience. Backscatter, 14-20.

9. Vachon P.W., Campbell J.W.M., Bjerkelund C.A., Dobson F.W., Rey M.T. (1997) Ship detection by the RADARSAT SAR: Validation of detection model predictions. Canadian Journal of Remote Sensing, 23(1), 48-59.

10. Liu C., Vachon P.W., Geling G.W. (2005) Improved Ship Detection with Airborne Polarimetric SAR data. Canadian Journal of Remote Sensing, 31, 122-131.

11. Greidanus H., Lemoine G., Kourti N (2005) Satellite ship detection for fishery control. NURC, international symposium Remote Sensing Applications to support NATO Expeditionary, Operations, Lerici, Italy.

12. Willhauck, G., Caliz, J. J., Hoffmann, C., Lingenfelder, I. \& Heynen, M. (2005) Objectoriented ship detection from VHR satellite images. Semana Geomática. Barcelona, Spain.

13. Smith, F.W., Wright, M.H., 1971. Automatic ship photo interpretation by the method of moments. IEEE Trans. Comput. 20, 1089-1094.

14. TERRA (2003) http://terra.nasa.gov.

15. Fujisada, H. (1998) ASTER Level 1 data processing algorithm. IEEE Transactions on Geoscience \& Remote Sensing, 36, 1101-1112.

16. Abrams M., Hook S., Ramachandran B. (2002) Aster user guide, ver. 2. EROS data center, Sioux Falls. 52 p.

17. ASTER (2003) http://asterweb.jpl.nasa.gov/

18. Eastman R. (1999) Guide to GIS and image processing (volume 2). Clark Labs, Worchester, MA, $169 \mathrm{p}$.

19. Mather, P.M. (1987) Computer processing of remotely-sensed images. John Wiley and Son, New York, 352 pp.

20. Chienand C.H., Aggarwal J.K. (1984) A Normalized Quadtree Representation. Computer Vision, Graphics, and Image Processing, 26(3), 331-346.

21. Shaffer C.A., Samet H. (1987) Optimal Quadtree Construction Algorithms. Computer Vision, Graphics, and Image Processing, 37(3), 402-419.

22. Meng Jun-Min, TAO Ming-de, Zhang jie (2001) Remote sensing methods for ship wake detection in SAR image. Journal of Hydrodynamics, 13(3).

23. Garzelli, A. (1995) Detection of ship wakes in SAR images using morphological operators.

Computers \& Geosciences, 21(10), 1201-1203.

24. Burrough P. (1986) Principles of Geographical Information Systems. Oxford University Press, New York, 194 p.

25. Pitas, I. (1993) Digital image processing algorithms. Prentice Hall, London, 362 pp.

26. Miliaresis G., Kokkas N. (2007) Segmentation \& Object Based Classification for the Extraction of the Building Class from LIDAR DEMs. Computers \& Geosciences, 33, 10761087.

27 SPSS (1997) SPSS Base 7.5 Applications Guide, SPSS Inc. USA. 339 p.

28. Clark W., Hosking P. (1986) Statistical methods for geographers. John Wiley \& Sons, New York, $518 \mathrm{p}$.

29. Shaw G., Wheeler D. (1985) Statistical techniques in geographical analysis. John Wiley \& Sons, Chichester, $364 \mathrm{p}$.

30 Greidanus H. (2005) Assessing the operationality of ship detection from space. Proceedings of the Symposium on New Space Services for Maritime Users: The Impact of Satellite Technology on Maritime Legislation, UNESCO Headquarters, Paris, France. 


\section{TABLES}

Table 1. Objects parametric representation on the basis of their size (in pixels) and the mean reflectance in ASTER VNIR bands 1, 2 and 3. The fifth column indicates the cluster to which an each object belong.

\begin{tabular}{|c|c|c|c|c|c|}
\hline $\begin{array}{c}\text { Object } \\
\text { ID }\end{array}$ & Size & B 1 & B2 & B3 & cluster \\
\hline $\mathbf{1}$ & 114 & 27.6 & 49.5 & 18.7 & 1 \\
\hline $\mathbf{2}$ & 65 & 27.1 & 42.6 & 11.0 & 2 \\
\hline $\mathbf{3}$ & 56 & 27.7 & 42.1 & 17.2 & 1 \\
\hline $\mathbf{4}$ & 21 & 26.6 & 49.8 & 12.0 & 2 \\
\hline $\mathbf{5}$ & 34 & 54.3 & 53.7 & 13.9 & 5 \\
\hline $\mathbf{6}$ & 17 & 27.1 & 45.3 & 11.2 & 2 \\
\hline $\mathbf{7}$ & 27 & 28.3 & 40.8 & 13.0 & 2 \\
\hline $\mathbf{8}$ & 19 & 28.4 & 46.7 & 12.6 & 2 \\
\hline $\mathbf{9}$ & 82 & 26.4 & 13.7 & 17.8 & 3 \\
\hline $\mathbf{1 0}$ & 24 & 28.4 & 38.1 & 12.0 & 2 \\
\hline $\mathbf{1 1}$ & 27 & 27.4 & 19.4 & 11.3 & 4 \\
\hline $\mathbf{1 2}$ & 23 & 29.9 & 30.3 & 12.2 & 2 \\
\hline $\mathbf{1 3}$ & 141 & 27.0 & 14.2 & 18.3 & 3 \\
\hline $\mathbf{1 4}$ & 200 & 30.9 & 13.5 & 19.5 & 3 \\
\hline $\mathbf{1 5}$ & 23 & 27.0 & 28.0 & 11.0 & 2 \\
\hline $\mathbf{1 6}$ & 160 & 26.0 & 14.5 & 19.6 & 3 \\
\hline $\mathbf{1 7}$ & 2 & 28.5 & 54.5 & 10.5 & 2 \\
\hline $\mathbf{1 8}$ & 16 & 27.4 & 14.6 & 11.1 & 4 \\
\hline $\mathbf{1 9}$ & 56 & 28.6 & 14.6 & 13.2 & 4 \\
\hline $\mathbf{2 0}$ & 72 & 26.3 & 13.5 & 17.8 & 3 \\
\hline $\mathbf{2 1}$ & 107 & 26.8 & 13.8 & 17.6 & 3 \\
\hline $\mathbf{2 2}$ & 98 & 28.1 & 16.1 & 16.9 & 3 \\
\hline $\mathbf{2 3}$ & 114 & 26.7 & 13.9 & 14.9 & 3 \\
\hline $\mathbf{2 4}$ & 173 & 25.9 & 13.0 & 21.1 & 3 \\
\hline $\mathbf{2 5}$ & 56 & 26.5 & 15.3 & 13.1 & 4 \\
\hline $\mathbf{2 6}$ & 205 & 25.9 & 12.8 & 21.6 & 3 \\
\hline $\mathbf{2 7}$ & 98 & 24.9 & 12.5 & 15.3 & 3 \\
\hline $\mathbf{2 8}$ & 50 & 25.7 & 12.9 & 11.8 & 4 \\
\hline $\mathbf{2 9}$ & 19 & 25.9 & 13.4 & 10.1 & 4 \\
\hline $\mathbf{3 0}$ & 32 & 25.4 & 12.9 & 11.4 & 4 \\
\hline $\mathbf{3 1}$ & 61 & 25.0 & 12.4 & 12.5 & 4 \\
\hline $\mathbf{3 2}$ & 164 & 24.9 & 12.5 & 19.9 & 3 \\
\hline $\mathbf{3 3}$ & 184 & 27.0 & 13.0 & 18.7 & 3 \\
\hline $\mathbf{3 4}$ & 171 & 24.8 & 12.7 & 19.3 & 3 \\
\hline $\mathbf{3 5}$ & 189 & 24.6 & 12.2 & 19.4 & 3 \\
\hline $\mathbf{3 6}$ & 31 & 25.5 & 12.8 & 12.5 & 4 \\
\hline $\mathbf{3 7}$ & 43 & 25.6 & 13.2 & 12.5 & 4 \\
\hline $\mathbf{3 8}$ & 57 & 25.6 & 13.1 & 12.9 & 4 \\
\hline $\mathbf{3 9}$ & 102 & 24.6 & 12.3 & 16.0 & 3 \\
\hline $\mathbf{4 0}$ & 74 & 24.7 & 12.7 & 18.8 & 3 \\
\hline $\mathbf{4 1}$ & 56 & 24.1 & 12.3 & 11.8 & 4 \\
\hline $\mathbf{4 2}$ & 86 & 24.2 & 12.1 & 13.2 & 4 \\
\hline $\mathbf{4 3}$ & 14 & 26.4 & 53.1 & 13.4 & 2 \\
\hline $\mathbf{4 4}$ & 12 & 65.4 & 57.0 & 11.7 & 5 \\
\hline $\mathbf{4 5}$ & 12 & 26.1 & 50.0 & 10.9 & 2 \\
\hline $\mathbf{4 6}$ & 33 & 26.3 & 55.4 & 12.1 & 2 \\
\hline $\mathbf{4 7}$ & 28 & 26.3 & 38.6 & 12.1 & 2 \\
\hline $\mathbf{4 8}$ & 29 & 26.2 & 22.4 & 11.5 & 4 \\
\hline $\mathbf{4 9}$ & 16 & 26.4 & 35.9 & 11.6 & 2 \\
\hline $\mathbf{5 0}$ & 2 & 26.5 & 13.0 & 11.5 & 4 \\
\hline $\mathbf{5 1}$ & 16 & 26.9 & 41.5 & 11.1 & 2 \\
\hline $\mathbf{5 3}$ & 25 & 24.8 & 13.0 & 10.2 & 4 \\
\hline $\mathbf{5 4}$ & 20 & 24.3 & 12.1 & 11.6 & 4 \\
\hline $\mathbf{5 5}$ & 16 & 24.4 & 11.9 & 10.8 & 4 \\
\hline & & & & & \\
\hline
\end{tabular}

\begin{tabular}{|c|c|c|c|c|c|}
\hline $\begin{array}{c}\text { Object } \\
\text { ID }\end{array}$ & Size & B 1 & B2 & B3 & cluster \\
\hline $\mathbf{5 6}$ & 30 & 24.6 & 12.0 & 9.8 & 4 \\
\hline $\mathbf{5 7}$ & 74 & 25.2 & 14.0 & 14.3 & 4 \\
\hline $\mathbf{5 8}$ & 42 & 25.0 & 12.9 & 12.4 & 4 \\
\hline $\mathbf{5 9}$ & 16 & 24.5 & 11.9 & 11.8 & 4 \\
\hline $\mathbf{6 0}$ & 31 & 24.1 & 12.1 & 10.6 & 4 \\
\hline $\mathbf{6 1}$ & 82 & 25.0 & 12.3 & 14.1 & 4 \\
\hline $\mathbf{6 2}$ & 62 & 24.7 & 12.6 & 12.9 & 4 \\
\hline $\mathbf{6 3}$ & 53 & 24.0 & 12.0 & 13.3 & 4 \\
\hline $\mathbf{6 4}$ & 40 & 25.4 & 12.3 & 11.7 & 4 \\
\hline $\mathbf{6 5}$ & 61 & 24.8 & 12.2 & 12.6 & 4 \\
\hline $\mathbf{6 6}$ & 24 & 24.6 & 12.1 & 9.9 & 4 \\
\hline $\mathbf{6 7}$ & 38 & 23.9 & 11.8 & 10.7 & 4 \\
\hline $\mathbf{6 8}$ & 36 & 23.6 & 11.9 & 11.0 & 4 \\
\hline $\mathbf{6 9}$ & 98 & 24.2 & 12.0 & 13.5 & 4 \\
\hline $\mathbf{7 0}$ & 31 & 23.5 & 11.6 & 11.2 & 4 \\
\hline $\mathbf{7 1}$ & 73 & 23.8 & 11.6 & 11.6 & 4 \\
\hline $\mathbf{7 2}$ & 46 & 23.8 & 11.8 & 11.5 & 4 \\
\hline $\mathbf{7 3}$ & 40 & 24.0 & 12.1 & 11.1 & 4 \\
\hline $\mathbf{7 4}$ & 63 & 24.2 & 11.9 & 13.1 & 4 \\
\hline $\mathbf{7 5}$ & 64 & 23.6 & 11.6 & 11.0 & 4 \\
\hline $\mathbf{7 6}$ & 54 & 23.9 & 12.0 & 11.8 & 4 \\
\hline $\mathbf{7 7}$ & 24 & 24.2 & 11.8 & 9.4 & 4 \\
\hline $\mathbf{7 8}$ & 23 & 23.8 & 11.8 & 13.3 & 4 \\
\hline $\mathbf{7 9}$ & 52 & 24.1 & 11.8 & 13.1 & 4 \\
\hline $\mathbf{8 0}$ & 36 & 24.0 & 12.0 & 11.4 & 4 \\
\hline $\mathbf{8 1}$ & 28 & 24.6 & 11.9 & 10.8 & 4 \\
\hline $\mathbf{8 2}$ & 80 & 23.8 & 11.8 & 12.4 & 4 \\
\hline $\mathbf{8 3}$ & 118 & 23.2 & 11.6 & 15.8 & 3 \\
\hline $\mathbf{8 4}$ & 101 & 24.0 & 11.8 & 12.9 & 4 \\
\hline $\mathbf{8 5}$ & 36 & 23.7 & 11.4 & 12.3 & 4 \\
\hline $\mathbf{8 6}$ & 61 & 24.2 & 12.0 & 12.8 & 4 \\
\hline $\mathbf{8 7}$ & 97 & 23.6 & 11.4 & 14.7 & 4 \\
\hline $\mathbf{8 8}$ & 107 & 23.7 & 11.9 & 14.1 & 4 \\
\hline $\mathbf{8 9}$ & 70 & 24.0 & 12.0 & 11.9 & 4 \\
\hline $\mathbf{9 0}$ & 107 & 23.8 & 11.9 & 13.5 & 4 \\
\hline $\mathbf{9 1}$ & 120 & 23.6 & 12.9 & 14.8 & 3 \\
\hline $\mathbf{9 2}$ & 81 & 23.6 & 11.6 & 12.0 & 4 \\
\hline $\mathbf{9 3}$ & 82 & 23.8 & 11.7 & 13.7 & 4 \\
\hline $\mathbf{9 4}$ & 46 & 24.0 & 11.9 & 10.4 & 4 \\
\hline $\mathbf{9 5}$ & 79 & 23.5 & 11.8 & 11.5 & 4 \\
\hline $\mathbf{9 6}$ & 110 & 23.6 & 11.8 & 13.6 & 4 \\
\hline $\mathbf{9 7}$ & 47 & 24.0 & 11.8 & 13.0 & 4 \\
\hline $\mathbf{9 8}$ & 181 & 23.9 & 11.9 & 15.1 & 3 \\
\hline $\mathbf{9 9}$ & 67 & 23.3 & 12.0 & 11.6 & 4 \\
\hline $\mathbf{1 0 0}$ & 72 & 23.4 & 11.9 & 15.0 & 4 \\
\hline $\mathbf{1 0 1}$ & 100 & 22.8 & 11.2 & 14.0 & 4 \\
\hline $\mathbf{1 0 2}$ & 28 & 23.9 & 11.5 & 9.8 & 4 \\
\hline $\mathbf{1 0 3}$ & 40 & 33.5 & 27.2 & 12.9 & 2 \\
\hline $\mathbf{1 0 4}$ & 61 & 25.8 & 12.9 & 14.6 & 4 \\
\hline $\mathbf{1 0 5}$ & 27 & 25.4 & 12.9 & 12.0 & 4 \\
\hline $\mathbf{1 0 6}$ & 179 & 24.9 & 12.4 & 20.9 & 3 \\
\hline $\mathbf{1 0 7}$ & 100 & 22.1 & 10.7 & 11.4 & 4 \\
\hline & & & & & \\
\hline
\end{tabular}


Table 2. The number of objects as well as, the gravity center (centroid) per cluster.

\begin{tabular}{|c|c|c|c|c|c|}
\hline \multirow{2}{*}{ Attributes } & \multicolumn{5}{|c|}{ Final Cluster Centers } \\
\cline { 2 - 6 } & $\mathbf{1}$ & $\mathbf{2}$ & $\mathbf{3}$ & $\mathbf{4}$ & $\mathbf{5}$ \\
\hline Size & 0.401 & -0.863 & 1.528 & -0.263 & -0.879 \\
\hline Band 1 & 0.335 & 0.340 & -0.036 & -0.283 & 6.662 \\
\hline Band 2 & 2.107 & 1.842 & -0.417 & -0.464 & 2.846 \\
\hline Band 3 & 1.602 & -0.525 & 1.648 & -0.439 & -0.212 \\
\hline $\begin{array}{c}\text { Number of objects in } \\
\text { each cluster }\end{array}$ & 2 & 16 & 21 & 66 & 2 \\
\hline
\end{tabular}

Table 3. Analysis of variance (ANOVA) for the centroid method.

\begin{tabular}{|c|c|c|c|c|c|c|}
\hline \multicolumn{7}{|c|}{ ANOVA } \\
\cline { 2 - 5 } & \multicolumn{2}{|c|}{ Cluster } & \multicolumn{2}{c|}{ Error } & \multirow{2}{*}{ Sig. } \\
\cline { 2 - 5 } & $\begin{array}{c}\text { Mean } \\
\text { Square }\end{array}$ & df $^{*}$ & $\begin{array}{c}\text { Mean } \\
\text { Square }\end{array}$ & df* & & \\
\hline Size & 16.845 & 4 & 0.379 & 102 & 44.493 & 0.000 \\
\hline Band 1 & 24.033 & 4 & 0.097 & 102 & 248.260 & 0.000 \\
\hline Band 2 & 24.295 & 4 & 0.086 & 102 & 281.348 & 0.000 \\
\hline Band 3 & 19.856 & 4 & 0.261 & 102 & 76.219 & 0.000 \\
\hline
\end{tabular}

The $\mathrm{F}$ tests should be used only for descriptive purposes because the clusters have been chosen to maximize the differences among cases in different clusters. The observed significance levels are not corrected for this and thus cannot be interpreted as tests of the hypothesis that the cluster means are equal.

*df: Degrees of fredom

Table 4. Distances between cluster centroids (divergence)

\begin{tabular}{|c|c|c|c|c|c|}
\hline \multicolumn{7}{|c|}{ Distances between Final Cluster Centers } \\
\hline Cluster & 1 & 2 & 3 & 4 & 5 \\
\hline 1 & 0 & & & & \\
\hline 2 & 2.487 & 0 & & & \\
\hline 3 & 2.789 & 3.960 & 0 & & \\
\hline 4 & 3.405 & 2.464 & 2.762 & 0 & \\
\hline 5 & 6.745 & 6.409 & 8.048 & 7.721 & 0 \\
\hline
\end{tabular}




\section{FIGURE CAPTIONS}

Fig. 1 ASTER VNIR band 1 image

Fig. 2 Quad tree based image decomposition

Fig. 3 Target rectangles of possible ship location and detail in ASTER VNIR band 3

Fig. 4 a) Resulted rectangles in stripped imagery, b) algorithm avoiding island regions

Fig. 5 left A portion of the slope image (note that pixels outside the rectangular regions of figure 3 were excluded), middle: The frequency histogram of the slope pixels within the rectangular regions of figure 3 . The $99.1 \%$ of the image pixels present slope $<10$, right: Selective objects segmented by image thresholding

Fig. 6 left Graphical representation of the cluster centroids (clusters 1 and 5 were excluded), right: spatial arrangement of the cluster 2 (the objects were enclosed in rectangular), cluster 3 (the objects were enclosed in circles) and cluster 4 superimposed over the ASTER VNIR band 1

Fig. 7 a) original ship patch, b) first snapshot, c) second snapshot, d) second snapshot with noise pixels removed, e) ship's principal eigenvector, f) direction through MBR centre and centre of gravity, $\mathbf{g}$ ) direction through snapshot comparison, h) direction through bright pixel

Fig. 8 Turning ship trace and convex hull

Fig. 9 a) Orientation of several ship patches, b) overall orientation statistic-traffic upon point of arrival, c) ship orientation and probable destinations

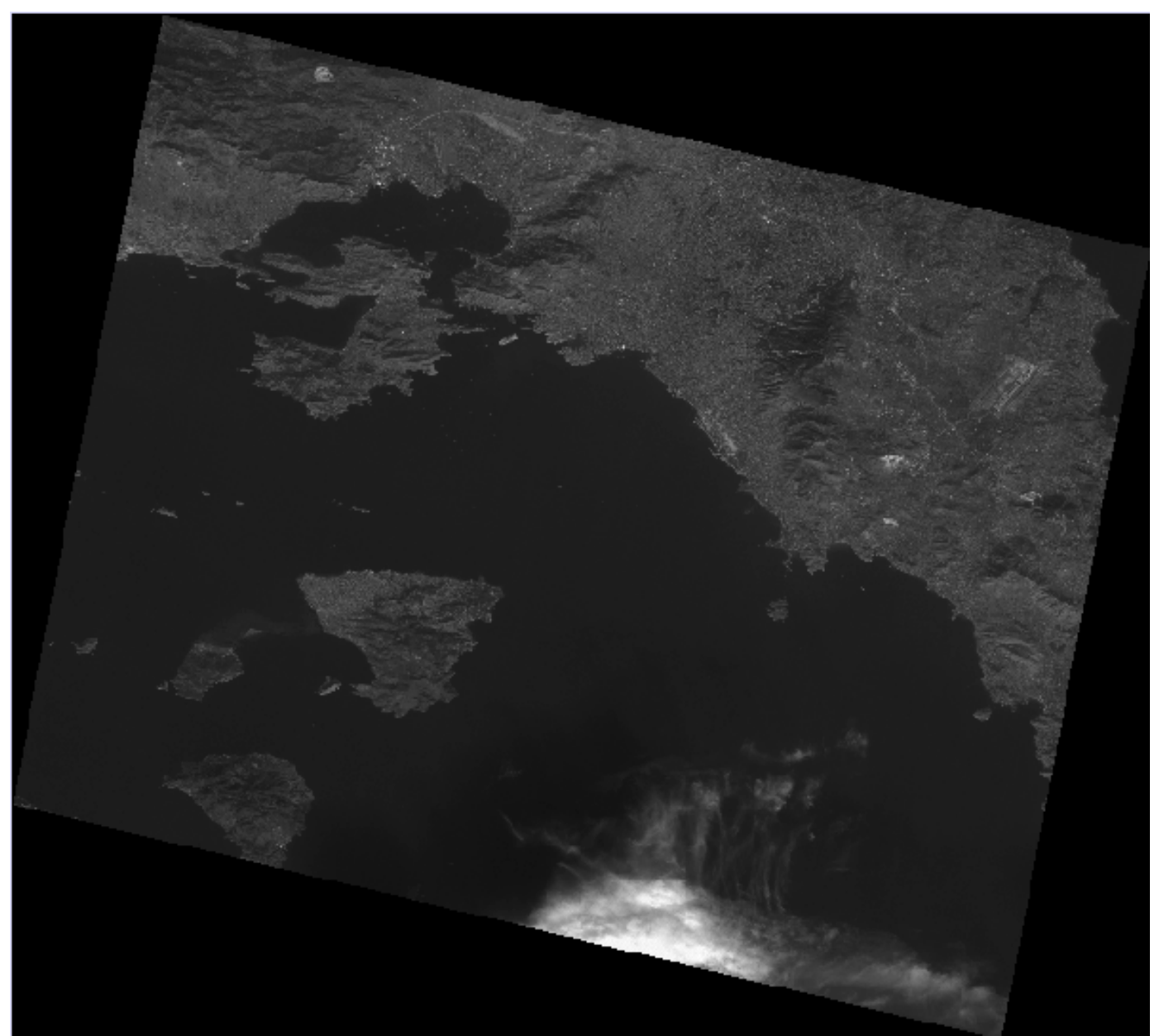

Figure 1 

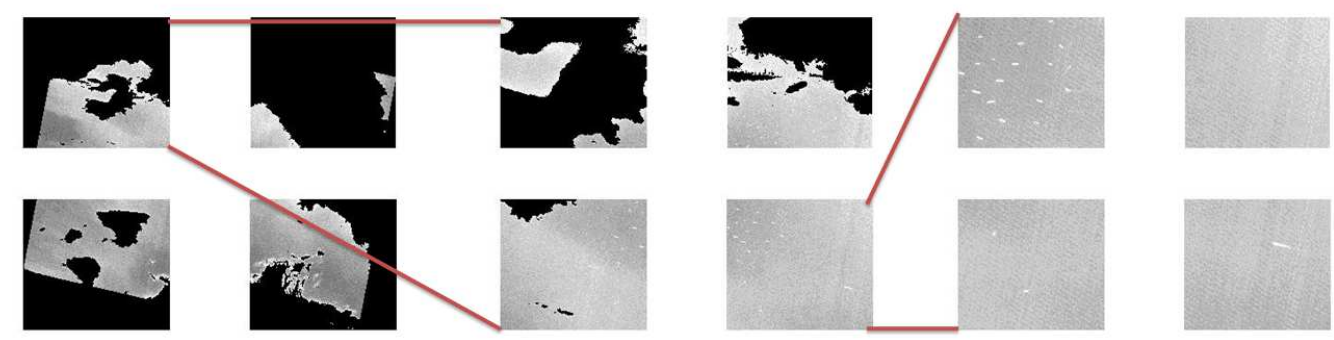

Figure 2

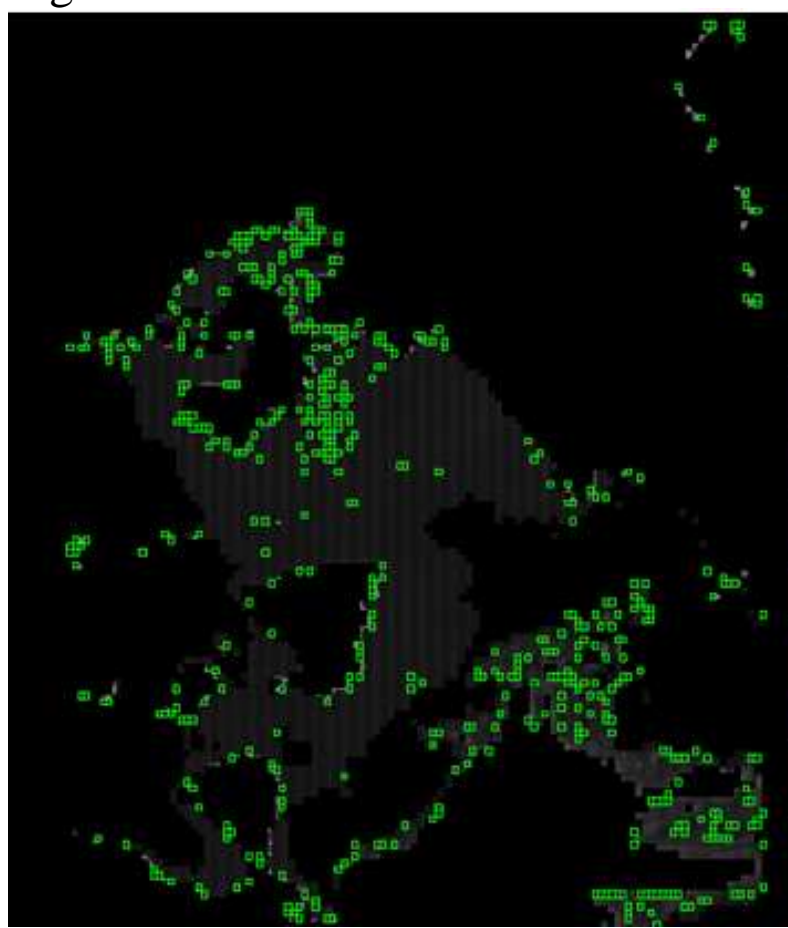

Figure 3

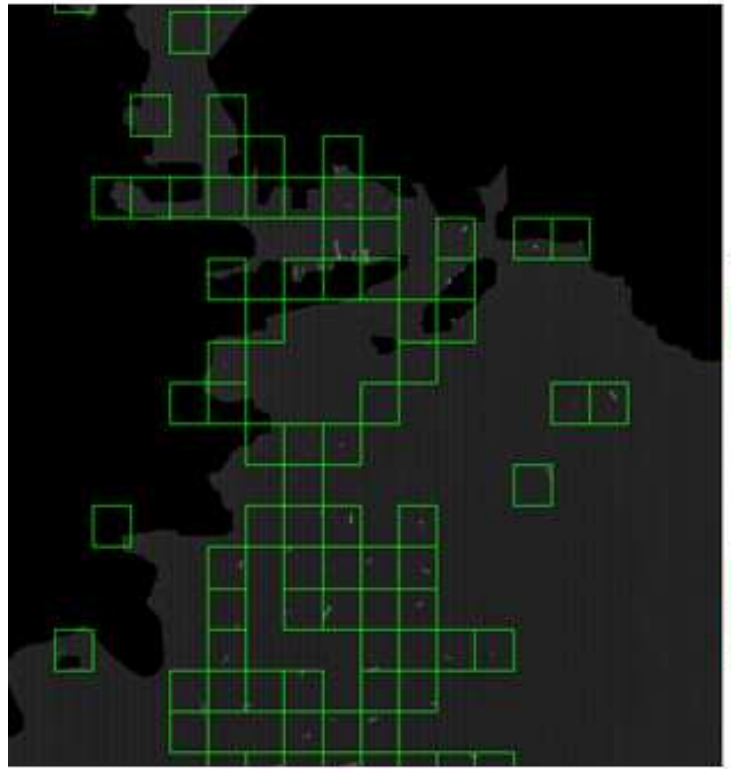

Figure 4 

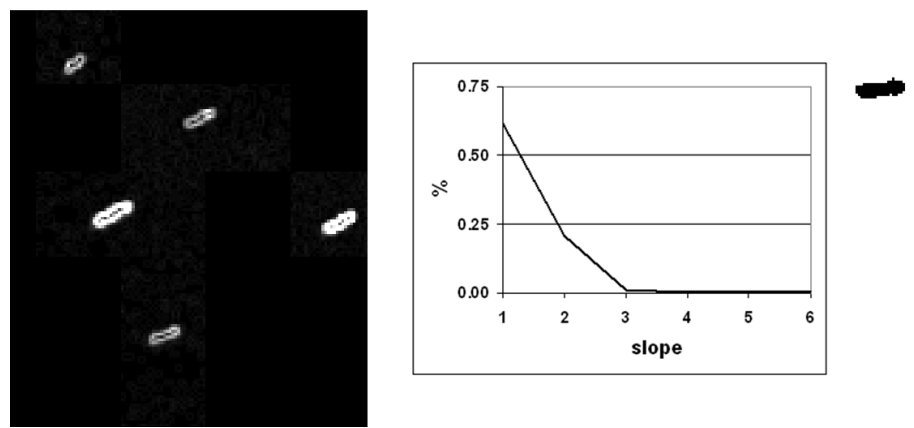

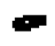

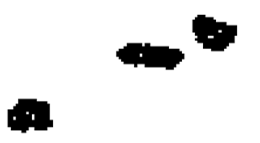

a

Figure 5

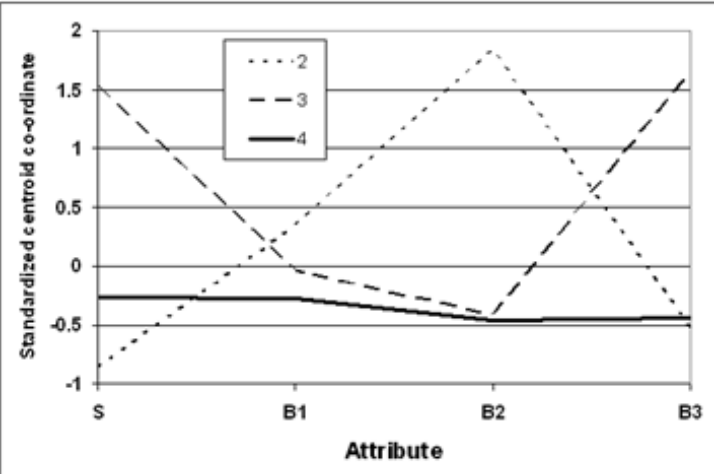

๑. $\therefore \therefore$

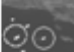

$-$

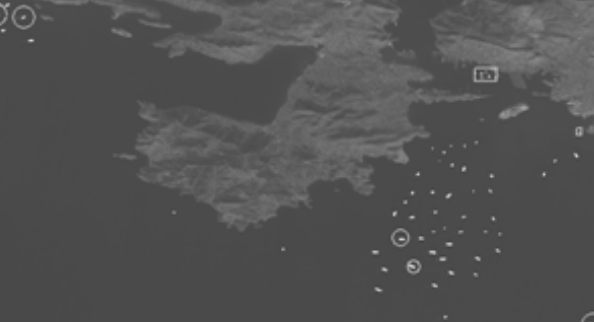

Figure 6
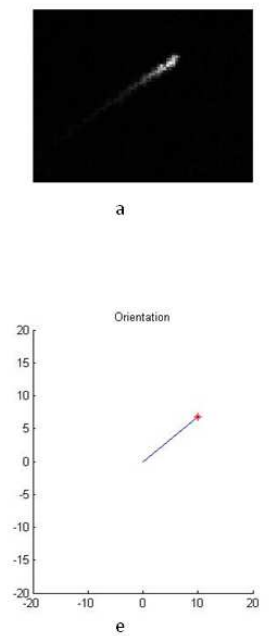

f
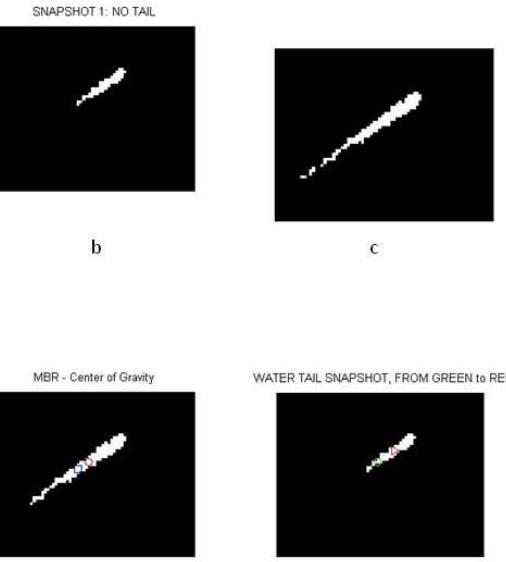

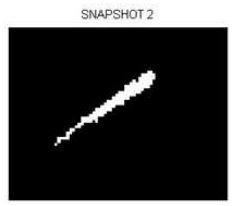

d

GPIGHTER PXELS, FROM GREEN to RED

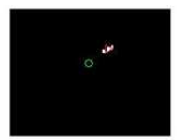

h

Figure 7
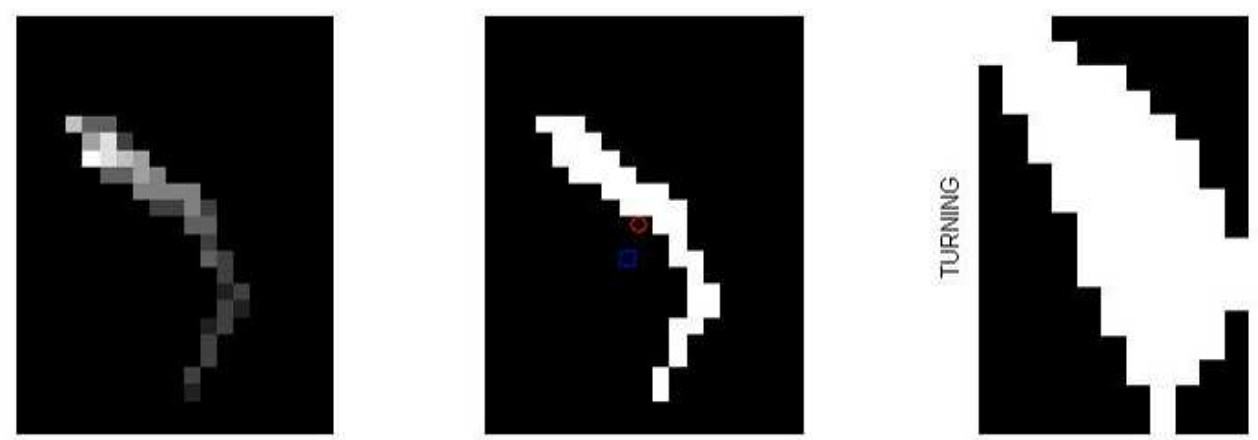

Figure 8 
